\title{
Prevalence of discordant elevations of state entropy and bispectral index in patients at amnestic sevoflurane concentrations: a historical cohort study \\ Fréquence des élévations discordantes de l'entropie basale et de l'index bispectral chez les patients à des concentrations de sévoflurane provoquant l'amnésie : une étude de cohorte historique
}

\author{
Richard H. Epstein, MD • Joni M. Maga, MD • Michael E. Mahla, MD • \\ Eric S. Schwenk, MD • Marc J. Bloom, MD, PhD \\ Received: 13 August 2017/Revised: 22 November 2017/Accepted: 6 February 2018/Published online: 9 March 2018 \\ (C) Canadian Anesthesiologists' Society 2018
}

\begin{abstract}
Background Processed electroencephalogram (EEG) monitors help assess the hypnotic state during general anesthesia or sedation. Maintaining the bispectral index (BIS) or state entropy (SE) between 40 and 60 has been recommended to mitigate anesthesia awareness. Nonetheless, SEs $>70$ were frequently observed at endtidal sevoflurane concentrations unlikely to allow awareness. We sought to determine the prevalence of elevated discordant measurements during BIS and SE monitoring.

Methods Electronic data collected over 11 months at two academic hospitals were retrospectively reviewed. At the hospital using SE, all cases were included with patients $\geq$ $18 \mathrm{yr}$ and sevoflurane administered for at least $30 \mathrm{~min}$ during surgery. A cohort of cases propensity matched by
\end{abstract}

This article is accompanied by an editorial. Please see Can J Anesth 2018; 65: this issue.

Electronic supplementary material The online version of this article (https://doi.org/10.1007/s12630-018-1085-3) contains supplementary material, which is available to authorized users.

R. H. Epstein, MD ( $₫)$ · J. M. Maga, MD

M. J. Bloom, MD, PhD

Department of Anesthesiology, Perioperative Medicine and Pain Management, Miller School of Medicine, University of Miami, 1400 NW 12th Avenue, Suite 3075, Miami, FL 33136, USA

e-mail: repstein@med.miami.edu

M. E. Mahla, MD · E. S. Schwenk, MD

Department of Anesthesiology, Sidney Kimmel Medical

College, Thomas Jefferson University, Philadelphia, PA, USA age and American Society of Anesthesiologist Physical Status were selected from the hospital using BIS. Elevated discordant EEG indices were defined as values $>70$ occurring during stable end-tidal sevoflurane concentrations $>1.5 \%$. The odds ratio $(\mathrm{OR})$ based on the probability of a case having at least one elevated discordant $S E$ or BIS lasting $\geq$ two minutes (primary endpoint) was calculated.

Results At each hospital, 3,690 cases were studied. The mean (95\% confidence interval [CI]) incidence of cases with at least one interval of an elevated discordant EEG index lasting at least two minutes was $3.6 \%$ (2.8\% to $4.4 \%)$ for SE compared with $0.24 \%(0.17 \%$ to $0.27 \%)$ for BIS (pooled OR, 17.0; 95\% CI, 8.3 to 34.7; $P<0.001$ ).

Conclusions The prevalence of an elevated discordant EEG index is much greater with SE than with BIS. Elevated index values occurring at anesthetic concentrations well above the awareness threshold need to be assessed to determine if they indicate an inadequate depth of anesthesia requiring treatment or if they simply reflect the underlying monitoring algorithm.

\section{Résumé \\ Contexte Les moniteurs d'électroencéphalographie (EEG) dont les données ont été analysées contribuent à évaluer l'état hypnotique pendant l'anesthésie générale ou la sédation. Le maintien de l'index bispectral (BIS) ou de l'entropie basale (SE, pour state entropy) entre 40 et 60 a été recommandé pour réduire l'incidence de l'éveil peropératoire. Toutefois, des entropies basales $>70$ ont fréquemment été observées à des concentrations télé-}


expiratoires de sévoflurane peu susceptibles de permettre un éveil. Nous avons tenté de déterminer la prévalence de mesures discordantes élevées pendant le monitorage du BIS et de l'entropie basale.

Méthode Les données électroniques colligées sur une période de 11 mois dans deux hôpitaux universitaires ont été rétrospectivement passées en revue. Dans l'hôpital utilisant l'entropie basale comme mesure, tous les cas de patients $\geq 18$ ans et auxquels on avait administré du sévoflurane pour un minimum de $30 \mathrm{~min}$ pendant la chirurgie ont été inclus. Une cohorte de propension de cas appariés selon l'âge et le système de classification du statut physique de l'American Society of Anesthesiologists (ASA) a été sélectionnée parmi les données colligées dans un autre hôpital utilisant le BIS. On a défini les indices d'EEG discordants élevés tels que des valeurs $>70$ survenant durant des concentrations télé-expiratoires stables de sévoflurane $>1,5 \%$. Le rapport de cotes $(R C)$ fondé sur la probabilité d'un cas ayant au moins une valeur d'entropie basale ou de BIS discordante élevée $\geq$ deux minutes (critère d'évaluation principal) a été calculé.

Résultats Au total, 3690 cas ont été étudiés dans chaque hôpital. L'incidence de cas dans l'intervalle de confiance (IC) à $95 \%$ moyen présentant au moins un intervalle d'un indice d'EEG discordant élevé durant au moins deux minutes était de 3,6\% (2,8\% à 4,4\%) lorsque l'entropie basale a été utilisée comme mesure, par rapport à $0,24 \%$ $(0,17 \%$ à $0,27 \%)$ lorsqu'on a utilisé le BIS (RC groupé, 17,0; IC à $95 \%, 8,3$ à 34,7; $P<0,001)$.

Conclusion La prévalence d'un indice d'EEG discordant élevé est bien plus importante avec l'entropie basale qu'avec le BIS. Des valeurs d'indice élevées survenant avec des concentrations anesthésiques bien au dessus du seuil d'éveil doivent être évaluées afin de déterminer si elles indiquent une profondeur d'anesthésie inadaptée qui nécessite un traitement ou si elles reflètent simplement l'algorithme de monitorage sous-jacent.

Various commercial monitors that signal process the electroencephalogram (EEG) are available to help assess the hypnotic state during general anesthesia (GA). ${ }^{1}$ At the University of Miami Hospital (UMH), E-entropy modules (GE Healthcare, Helsinki, Finland) are provided in all anesthetizing locations. The UMH providers customarily use this technology during GA in an effort to mitigate the occurrence of "anesthesia awareness," despite controversy regarding the effectiveness of such monitoring. ${ }^{2}$

The E-entropy module computes the Shannon entropy (a measure of information content) of the EEG waveform filtered to include only frequencies within a given range. ${ }^{3}$
Fundamentally, the more complex the waveform is, the higher the entropy. The module produces two values: 1 ) reactive entropy (RE), measured between 0.8 and $47 \mathrm{~Hz}$, and 2) state entropy (SE), measured between $0.8 \mathrm{~Hz}$ and 32 Hz. Frequencies outside the range of interest are removed using high- and low-pass filters. The RE is always $\geq \mathrm{SE}$ because RE includes frequency components between 32 and $47 \mathrm{~Hz}$, mostly contributed by electromyographic (EMG) activity. The SE, an analog to BIS, was described by Viertiö-Oja et al. in their technical description of the entropy algorithm as "a stable indicator of the effect of hypnotics on the cortex." 4 Although SE is scaled from 091, the manufacturer recommends titrating the anesthetic to maintain the SE between 40-60 during $\mathrm{GA}^{5}{ }^{5}$ the same range recommended for BIS, which is scaled from $0-100 .{ }^{6}$ According to the departmental guidelines, anesthesia providers at $\mathrm{UMH}$ are instructed to titrate the anesthetic to maintain $\mathrm{SE}<60$ to mitigate the risk of awareness, based on data that suggested the rapid return of responsiveness to command after a propofol bolus dose once the BIS had moved above $65 .^{7}$

Because the EMG signal can have frequency components $<32 \mathrm{~Hz}^{8}$ and entropy is a mathematically defined quantity, we were concerned that SE might not accurately reflect the degree of hypnosis. Indeed, as the use of entropy monitoring expanded at $\mathrm{UMH}$, discordance between elevated values of SE and the apparent clinical state of patients began to be noted frequently. These were not transient episodes related to transitions from consciousness to unconsciousness, as previously described for $\mathrm{SE},{ }^{9}$ but rather occurred intraoperatively during the administration of stable anesthetic concentrations of sevoflurane or propofol (available as Electronic Supplementary Material [ESM], Appendix B, Fig. S3). Despite the lack of somatic or sympathetic manifestations of inadequate anesthesia, providers often gave additional anesthetics or analgesics because of concern about possible awareness. Examination of the raw EEG waveform often showed EMG activity (available as ESM, Appendix C, Fig. S4).

The aim of this study was to determine if discordant elevations of the processed EEG index (i.e., an EEG index value in the range of potential awareness occurring at a sevoflurane concentration considered extremely unlikely to result in awareness) were more common with SE monitoring $v s$ BIS monitoring. For convenience, we subsequently refer to discordant SE or BIS (rather than discordant "elevations" of the EEG index), meaning an elevation in the index that is incongruous with the clinical anesthetic state.

The clinical occurrence of discordant SE values and the potential implications of administering additional anesthesia to patients who were already adequately 
anesthetized motivated us to perform the current study. Such interventions might lead to additional expense from the administration of unnecessary anesthetics, prolonged emergence, and excessive opioid-induced respiratory depression. We tested the hypothesis that the prevalence of discordant SE would be greater than discordant BIS at stable sevoflurane concentrations $>1.5 \%$. Our primary endpoint was the odds ratio (OR) for the probability of a discordant value of the processed EEG value lasting $\geq$ two minutes (i.e., prolonged, not transient elevations).

\section{Methods}

The Institutional Review Boards at the University of Miami (Institutional Review Board ID\# 20170158 on 4 April 2017) and Thomas Jefferson University (Control \#17D.149 on 27 March 2017) approved this historical cohort study as exempt from requiring written patient consent. The Strengthening the Reporting of Observational Studies in Epidemiology (STROBE) checklist was followed by this study report. ${ }^{10}$

\section{Data collection}

During the study interval, there was exclusive use of entropy at UMH and BIS at Thomas Jefferson University Hospital (TJUH). Data from all UMH adult (age $\geq 18 \mathrm{yr}$ ) cases performed between 14 March 2016 and 8 February 2017 were extracted from the anesthesia information management system (AIMS) database (Innovian ${ }^{\circledR}$, Dräger, Telford, PA, USA). The start date corresponded to the introduction of entropy monitoring into the operating room. For each case, we determined the patient's age, American Society of Anesthesiologists Physical Status (ASA-PS), gender, date of surgery, case duration (defined as the interval between entering and leaving the operating room), surgical interval (defined as the difference between the surgery begin and end times), total dose of each nondepolarizing neuromuscular blocking drug, total dose of opioids, as well as all values of SE, RE, and end-tidal sevoflurane concentrations (nominally recorded at oneminute intervals). Opioids were converted into fentanyl $\mu \mathrm{g}$ equivalents by the following conversion factors: $10 \mu \mathrm{g}$ fentanyl/1 $\mathrm{mg}$ morphine; $50 \quad \mu \mathrm{g}$ fentanyl/1 $\mathrm{mg}$ hydromorphone; $10 \mu \mathrm{g}$ fentanyl/1 $\mu \mathrm{g}$ sufentanil; $2 \mu \mathrm{g}$ fentanyl $/ 1 \mu \mathrm{g}$ remifentanil.

Cases at UMH were included if the patient underwent GA with sevoflurane administered for at least $30 \mathrm{~min}$ during the interval between surgery begin and end, and entropy was monitored. Only sevoflurane cases were included because this agent was administered in $>95 \%$ of all cases where a volatile anesthetic was used. Cases with total intravenous
GA maintained with propofol by continuous infusion were excluded because there was not an independent means to assess the depth of anesthesia, in contrast to end-tidal volatile agent monitoring. Also, the dose response curve for propofol is wide, ${ }^{11-13}$ compared with that of sevoflurane. ${ }^{14}$ Thus, even with pharmacokinetic/pharmacodynamic modeling of effect site concentrations, we would not have been able to be clearly interpret an elevated index as being discordant with the depth of anesthesia.

Because utilization of entropy monitoring at UMH during GA increased progressively from approximately $8 \%$ to $75 \%$ over the study interval, data were batched by date of surgery in deciles based on the sequential order of the cases rather than by equal date ranges. The increased use of entropy monitoring resulted from a departmental guideline to monitor all GA cases using a processed EEG device. All batches had the same number of cases, thus weighting them equally.

Bispectral index values were infrequently present in the AIMS database at UMH, as the external BIS monitors used prior to the introduction of entropy had not been electronically interfaced with the AIMS, and manual entries were infrequently and sporadically recorded. Thus, the BIS discordance data were derived from the TJUH site using equivalent inclusion criteria for cases as applied at UHM, with BIS being collected in place of SE along with the amount of EMG activity. Data from the selected cases as described above were retrieved from the AIMS (Innovian) at this institution (also, TJUH and UMH are both tertiary care academic hospitals with a similar mix of patients and surgical specialties). Because BIS was utilized in only $8 \%$ of cases receiving GA at TJUH (as opposed to $75 \%$ for SE at UMH), the date range for cases was expanded from 1 January 2014 to 8 February 2017 to allow better propensity matching to the UMH cases. At TJUH, BIS is recorded using the BISx Power Link ${ }^{\mathrm{TM}}$ module (Philips Medical Systems, The Netherlands). There was no departmental policy related to monitoring processed EEG during GA at TJUH.

Cases from both UMH and TJUH were sorted in reverse chronological order. Study cases from TJUH were selected by propensity matching based on the combination of ASAPS and quartile of age from the UMH data set: 18-47, 4858, 59-68, and $\geq 69 \mathrm{yr}$. Age and ASA-PS were selected for matching because the BIS and SE at which loss of consciousness occurs vary by age, ${ }^{15}$ and increasing ASAPS is associated with greater sensitivity to propofol. ${ }^{16}$ Matching at TJUH was conducted without replacement using a greedy algorithm, ${ }^{17}$ starting from the most recent case at UMH and working backward. There was no trend in either direction for the use of BIS at TJUH over the data range ( $P=0.99$, by the Mann-Kendall test). 


\section{Definition of discordant BIS or SE}

We defined a discordant BIS or SE as the occurrence of an index value $>70$ in the presence of a stable end-tidal sevoflurane concentration $>1.5 \%$. We selected a threshold index $>70$ to define discordance because these values would represent cause for clinical concern of awareness and potentially result in an intervention by the provider (e.g., administering more anesthetic). The end-tidal sevoflurane concentration threshold of $1.5 \%$ (i.e., approximately 0.75 minimum alveolar concentration $[\mathrm{MAC}])^{18}$ to define unconsciousness was deliberately conservative, as we wanted a value in the typical clinical range during the surgical part of the procedure extremely unlikely to be associated with awareness. ${ }^{19}$ Numerous studies have consistently found that MAC-awake for sevoflurane is approximately $0.33 \mathrm{MAC},{ }^{20,21}$ with a steep

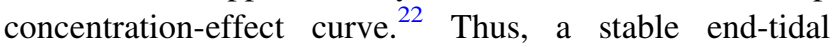
sevoflurane concentration of 0.75 MAC represents approximately 2.25 MAC-awake, a concentration at which responsiveness would be extremely unlikely and recall even less likely. The percentages of cases where there was at least one interval between the AIMS milestone events of surgery begin and surgery end lasting at least one, two, three, four, or five consecutive minutes with a discordant entropy reading were determined. Intervals with such values were only included if the difference between the inspired and end-tidal sevoflurane concentration was $\leq 0.2 \%$, representing a steady state, rather than the transition from one concentration to another, during which the brain concentration might lag behind the end-tidal concentration.

The chosen discordance threshold was also supported by two related studies. In a study by Flaishon et al. ${ }^{7}$ involving 12 subjects who received an induction dose of propofol, $60 \%$ were responsive to commands at BIS $\leq 70$. Similarly, in a study by Glass et al., ${ }^{23}$ approximately $50 \%$ of patients were responsive following isoflurane anesthesia at BIS of 70. We were unable to find any similar studies looking at the return of responsiveness in which entropy was monitored; nonetheless, we used the same threshold of 70 for SE based on the manufacturer's recommendations to titrate SE to the same range as BIS. ${ }^{5}$ Nonetheless, because the maximum value of SE is 91 , not 100 , the choice of 70 represents potential bias in favour of SE, because a lower threshold for SE might reasonably have been selected (i.e., increasing the prevalence of discordance).

Assessment of EMG influence on discordant SE and BIS

It is well known that EMG activity can spuriously increase the value of both BIS and SE. The amount of EMG activity is calculated by BIS monitors in units of $\mathrm{dB}$, but this is not reported by the GE entropy module. Rather, the amount of EMG activity was inferred from the difference between the RE and SE indices (i.e., and thus is a unitless measurement). This calculation is valid because there is little power (i.e., information) from the EEG in the frequency range from $32-47 \mathrm{~Hz}$; rather, the contribution to Shannon entropy in this range is contributed by facial muscle EMG. The interpretation of a difference between $\mathrm{SE}$ and RE is thus the presence of sympathetic activation (i.e., nociception resulting in increased frontalis motor activity). ${ }^{6}$

To assess the extent to which high levels of EMG activity might be contributing to a discordant elevation in $\mathrm{SE}$, we calculated the difference between RE and SE for each patient at each measurement time. For measurements when the end-tidal sevoflurane concentration was stable and $>1.5 \%$, we compared the RE-SE differences when the SE was $>70$ to when it was $<60$. We selected a threshold of 70 because values above this raise concern about the possibility of awareness, as described previously. Values $<60$ are in the range for both BIS and SE associated with an extremely low likelihood of awareness. To assess the extent to which high levels of EMG activity might be contributing to a discordant elevation in BIS, we similarly compared the EMG values when BIS was $>70$ to when it was $<60$.

Because the units of EMG activity measured by the BIS monitor are in $\mathrm{dB}$ and the amount inferred from the entropy module is unitless, a direct comparison between the amounts of EMG present between the two monitors could not be made. Rather, the assessment was made within the groups concerning the relative contribution of EMG to index values $<60$ and $>70$.

\section{Comparison of use of nondepolarizing neuromuscular blocking drugs}

The use of nondepolarizing neuromuscular blocking drugs (NMBDs) at each hospital was determined among all included cases, and the total dose for each NMBD (in $\mathrm{mg} \cdot \mathrm{min}^{-1}$ ) was determined and normalized by dividing the total dose by the case duration in minutes. Because rocuronium accounted for approximately 97\% NMBD at each hospital, only the doses of rocuronium were compared between cases monitored with SE or BIS. Quantitative assessment of the extent of neuromuscular blockade was available only at UMH, preventing a direct comparison with the values at TJUH. 
Statistical analysis

Based on an estimated prevalence of $2 \%$ for a discordant elevation in SE lasting at least two minutes, a pooled standard deviation (SD) of $1 \%$, and $\alpha=0.05$, a sample size of $n=$ ten batches would provide $90 \%$ power to detect an absolute difference of $1.5 \%$ between the two EEG indices using an unpaired two-sided Student $t$ test.

Cases were divided into ten batches (deciles) representing the sequential monitoring of patients at $\mathrm{UMH}$ and the matching cases at TJUH. Analysis using batch means ${ }^{24-29}$ accounts for the presence of unmeasured correlations among cases and also adjusts for potential nonGaussian distributions of values within the batches, the latter a statistical consequence of the central limit theorem. ${ }^{30}$

Comparisons between patients at UMH and TJUH for age in years, end-tidal sevoflurane concentration, minutes of processed EEG monitoring, minutes of sevoflurane exposure, processed EEG index (BIS or SE), normalized total rocuronium doses, total fentanyl equivalents $(\mu \mathrm{g})$, and percentage of patients with male gender were done using the method of batch means and the two-sided Student $t$ test. Assessment of the EMG contribution to discordant elevations in SE or BIS (as previously described) was done using batch means and the two-sided Student $t$ test. The percentages of cases in which there was at least one episode of a discordant index ( $>70$ ) lasting $\geq$ one minute, $\geq$ two minutes, $\geq$ three minutes, $\geq$ four minutes, and $\geq$ five minutes were compared between entropy and BIS using the Kruskal-Wallis test. Comparison of the distributions of ASA-PS between UMH and TJUH was made using the Chi-square test. The presence of trends was assessed using the Mann-Kendall test. Systat v13 (Systat Software, San Jose, CA, USA) was used for these statistical tests. For all tests, $P<0.01$ was required for statistical significance based on the Holm-Bonferroni adjustment for the number of relevant multiple comparisons. As sensitivity analyses, the prevalences of discordant SE and BIS lasting $\geq$ two minutes using a threshold index of $>50$ and $>55$ were also compared (similarly as described above using a threshold index of $>70$ ).

For calculation of the OR of a discordant elevation in the EEG index when using SE vs BIS, the Cochran-MantelHaenszel test was computed using the mantelhaen.test function in the R v3.4.1 stats library. ${ }^{31}$

\section{Results}

During the study interval, 3,690 cases were analyzed at $\mathrm{UMH}$; these were well matched to an additional 3,690 cases at TJUH for age, ASA-PS, gender, and duration of
EEG monitoring (Table). Providers at TJUH tended to run slightly lower end-tidal concentrations of sevoflurane than their counterparts at UMH (difference, $-0.17 \%$; $95 \% \mathrm{CI}$, 0.23 to $-0.96 \% ; P<0.001$; Table). Providers at TJUH also administered less opioid than their counterparts at UMH (difference, $-35 \mu \mathrm{g} ; 95 \% \mathrm{CI},-47$ to $-22 \mu \mathrm{g} ; P<0.001$; Table). There was no difference in the mean (SD) percentage of cases at the two hospitals where a nondepolaring muscle blocker was used [84 (1)\%, UMH vs 86 (1)\%, TJUH; $P=0.08$ ]. Higher mean (SD) normalized doses of rocuronium were administered at $\mathrm{UMH}$ than at TJUH [0.34 (0.01) $\mathrm{mg} \cdot \mathrm{min}^{-1}$ vs $0.27(0.01) \mathrm{mg} \cdot \mathrm{min}^{-1}$, respectively; $P<0.001$ ).

Prolonged episodes of discordant BIS readings $>70$ were rare. The mean (95\% confidence interval [CI]) incidence of cases with at least one interval of an elevated discordant EEG index lasting at least two minutes was $3.6 \%$ (95\% CI, $2.8 \%$ to $4.4 \%$ ) for $\mathrm{SE}$ compared with $0.24 \%$ (95\% CI, $0.17 \%$ to $0.27 \%$ ) for BIS (pooled OR, 17.0; 95\% CI, 8.3 to $34.7 ; P<0.001$ ). Accordingly, our primary hypothesis was supported. Furthermore, cases with a least one episode of a discordant EEG index lasting for all time periods from $\geq$ one to $\geq$ five minutes were more likely with SE than with BIS $(P<0.001$ for all comparisons; Fig. 1$)$.

The sensitivity analyses using discordant EEG index threshold values of 50 and 55 showed similar results. The episodes of the EEG index $>50$ lasting $\geq$ two minutes occurred in $11.1 \%$ (95\% CI, $8.0 \%$ to $10.2 \%$ ) of cases with SE vs $1.4 \%$ (95\% CI, $0.7 \%$ to $2.01 \%$ ) of cases with BIS (pooled OR, 9.1; 95\% CI, 6.8 to 12.3; $P<0.001$ ). Episodes of the EEG index $>55$ lasting $\geq$ two minutes occurred in $9.3 \%$ (95\% CI, $8.3 \%$ to $10.3 \%$ ) of cases with SE vs $1.0 \%$ (95\% CI, $0.3 \%$ to $1.75 \%$ ) of cases with BIS (pooled OR, 9.1; 95\% CI, 7.1 to $13.9 ; P<0.001)$.

As for the contribution of EMG to discordant elevations in the EEG indices, when the SE value was discordant (SE $>70$ and end-tidal sevoflurane concentration $>1.5 \%$ ), the mean (SD) amount of EMG (i.e., the difference between $\mathrm{RE}$ and SE) was $8.3(0.4), P<0.001$. In contrast, when the SE was appropriate (SE $<60$ and end-tidal sevoflurane concentration $>1.5 \%$ ), the mean (SD) amount of EMG was $2.5(0.1)(P<0.001$, for the pairwise differences in EMG among the ten batches). Similarly, when the BIS was discordant, the mean (SD) amount of EMG was larger [45.0 $(1.8) \mathrm{dB}]$ than when the BIS was appropriate [29.2 (0.3) dB; $P<0.001]$.

Appendix A (available as ESM) indicates that, overall, the values of BIS and SE were similar, as was their variability. What is of clinical relevance, however, is the outlier behaviour related to the prevalence of elevated index values discordant with the clinical state of the patient. 
Table Characteristics of cases with EEG monitoring and general anesthesia with sevoflurane

\begin{tabular}{|c|c|c|c|}
\hline & $\begin{array}{l}\mathrm{UMH} \\
n=10^{\mathrm{a}}\end{array}$ & $\begin{array}{l}\text { TJUH } \\
n=10^{\mathrm{b}}\end{array}$ & $P$ \\
\hline Patient age (yr), mean (SD) & $56.9(1.6)$ & $57.1(4.3)$ & 0.88 \\
\hline EEG monitoring $(\mathrm{min})$, mean $(\mathrm{SD})$ & $153.6(10.1)$ & $157.6(5.1)$ & 0.28 \\
\hline End-tidal sevoflurane, mean (SD) & $1.80(0.09)$ & $1.64(0.05)$ & 0.001 \\
\hline$\%$ Male, mean (SD) & $53.8 \%(1.4 \%)$ & $51.9 \%(4.0 \%)$ & 0.18 \\
\hline \multicolumn{4}{|l|}{ ASA Physical Status, $n(\%)$} \\
\hline I & $221(6.0 \%)$ & $214(5.8 \%)$ & \\
\hline II & $1,709(46.3 \%)$ & $1,716(46.5 \%)$ & \\
\hline III & $1,531(41.5 \%)$ & $1,531(41.5 \%)$ & \\
\hline IV & $219(5.9 \%)$ & $224(6.1 \%)$ & \\
\hline $\mathrm{V}$ & $10(0.3 \%)$ & $5(0.1 \%)$ & \\
\hline All ASA-PS & $3,690(100 \%)$ & $3,690(100 \%)$ & 0.76 \\
\hline
\end{tabular}

ASA-PS = American Society of Anesthesiologists Physical Status; EEG = electroencephalogram; SD = standard deviation; SE = state entropy; TJUH $=$ Thomas Jefferson University Hospital; UMH = University of Miami Hospital

${ }^{a}$ Cases were ordered consecutively and batched by decile

b Cases at TJUH were propensity matched to the sequential patients at UMH by ASA-PS and age group (see Methods)

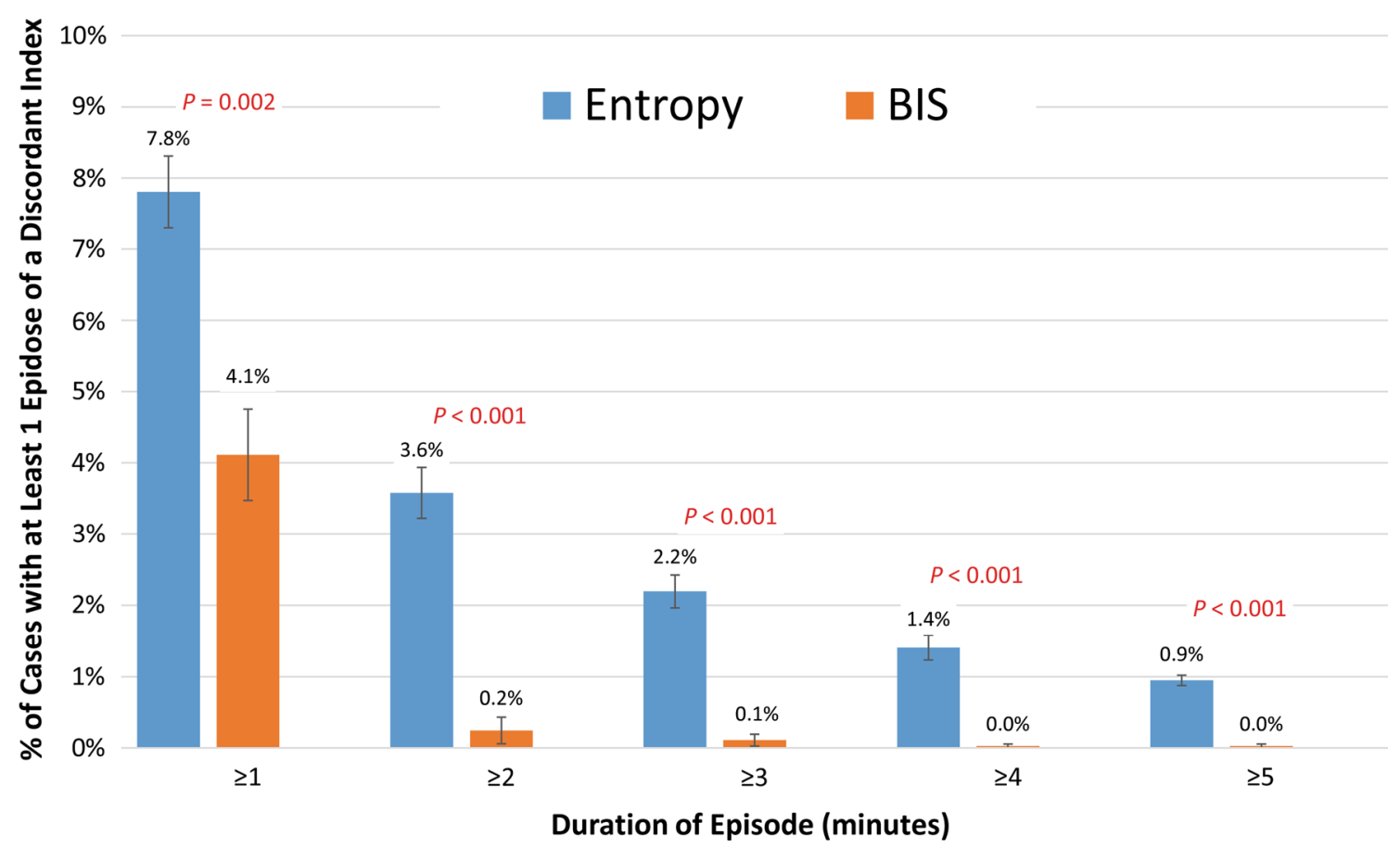

Figure 1 Percentages of cases with elevated discordant values of state entropy (SE) or bispectral index (BIS) for consecutive oneminute to five-minute durations. State entropy was measured using the E-entropy module (GE Healthcare, Helsinki, Finland) and BIS with the BISx Power Link ${ }^{\mathrm{TM}}$ module (Philips Medical Systems, The Netherlands). Discordant readings were those in which the index was $>70$ at the same time that the end-tidal sevoflurane concentration was $>1.5 \%$. Percentages of discordant SE and BIS were determined among cases in adults with EEG monitoring and general anesthesia with sevoflurane at the University of Miami Hospital (UMH) and Thomas Jefferson University Hospital (TJUH), respectively. Data were analyzed in decile batches, ordered sequentially by the date of surgery, with the mean and standard errors shown. Cases at TJUH were propensity matched to the UMH cases by patient age and American Society of Anesthesiologists Physical Status. $P<0.001$ for all comparisons at all time intervals 


\section{Discussion}

Our results indicate that there was a much higher intraoperative prevalence of prolonged discordant SE readings $(>70$ at stable end-tidal sevoflurane concentrations $>1.5 \%$ ) compared with those encountered during BIS monitoring. Measures of hypnosis that conflict with the perceived clinical state of the patient are problematic for two major reasons. First, such occurrences undermine confidence in the reliability of the monitor as an assessment of the anesthetic state. Second, if the monitor's reading is accepted, this can lead to the unnecessary administration of additional anesthetics or analgesics. Such episodes lasting at least two minutes were rare with BIS $(0.24 \%$ of cases) but approximately 17 times more common with SE (3.6\% of cases). To put this in perspective, if 50 cases were monitored daily, one would expect to see approximately one case with discordant BIS lasting two minutes every two weeks vs approximately two cases a day with entropy.

The greater prevalence of discordant SE cannot be explained by "lighter" levels of anesthesia administered at UMH because the providers there administered higher endtidal sevoflurane concentrations than their counterparts at TJUH. Neither can this discrepancy likely be explained by a lesser degree of analgesia because higher doses of opioids were administered at UMH than at TJUH. Finally, a greater degree of EMG activity during SE monitoring than with BIS was also not a likely explanation because larger doses of muscle relaxant were administered at UMH than at TJUH. If the two monitoring techniques were equivalent concerning the incidence of index values discordant with the clinical state of patients, each of these three differences would have been expected to bias the results toward less EMG at UMH. The implication of these differences is that if there were bias, it would be in the direction of greater sympathetic activity and consequently higher EMG activity at TJUH than at UMH.

While there are other sources of electrical noise in the OR that can interfere with processed EEG analysis, notably electrosurgical devices, these are in widespread use at both facilities. The differences cannot be explained by other sources of electrical activity in the OR such as Bluetooth or wireless communications. The reason is that the frequencies produced by those devices are in the 300 $\mathrm{kHz}$ and 1.2 MHz (typically $500 \mathrm{kHz})^{32}$ and 2.400 to 2.495 $\mathrm{GHz}$ range, ${ }^{33,34}$ respectively, and would have been filtered out by both devices.

A large component of the differential behaviour between SE and BIS therefore likely reflects a greater sensitivity to the effect of EMG on the calculation of SE. This finding is at odds with the representation that SE is reflective of the anesthetic state of the patient and that the difference between the RE and SE is indicative of the degree of sympathetic reactivity. ${ }^{5}$ BIS represents a nonlinear combination of weighted subparameters in the time, frequency, and bispectral domains, optimized against an extensive EEG library aligned with a concomitant assessment of the patients' sedation level. ${ }^{35}$ Thus, because the contribution of EMG activity to the BIS algorithm is less than with SE, it is perhaps not surprising that BIS is less subject to the effect of EMG. In contrast to BIS, SE is a mathematically derived parameter that only reflects the information content of the signal within a frequency domain spectrum from 0.8 to $32 \mathrm{HZ}$. This range includes a contribution by EMG activity $^{3}$; this adds information content, thus increasing the entropy of the processed signal. One cannot simply filter out EMG overlapping the EEG spectrum of interest, as this will also filter the real EEG signal, spuriously reducing the entropy. Unfortunately, unlike the BIS monitor, the current version of the E-entropy module does not display the amount of EMG present in the signal, which could serve as a guide in the interpretation of unexpected elevations of the SE. While large amounts of EMG activity are usually easily recognized in the raw waveform, lower levels of EMG may be difficult to detect visually, given the limited resolution of the screen display and the relative inexperience of most anesthesia providers in interpreting raw EEG signals.

Some studies comparing BIS and SE simultaneously, albeit in small numbers of patients, have shown statistically different values, ${ }^{8,36}$ in contrast with our findings (available as ESM, Fig. S1). Nonetheless, Pilge and Schnieder questioned the meaningfulness and relevance of such comparisons, in part because the indices are derived from different training sets, use different signal processing algorithms, may not express the same curvilinear relationship to the probability of consciousness, and lack a gold standard for reference comparison. ${ }^{37}$ Although our study of many thousands of patients of all ages showed that SE and BIS produced values that were not different over a range of clinically relevant end-tidal sevoflurane concentrations (available as ESM, Fig. S1), the much higher prevalence of discordant SE appears to be a fundamental difference in the clinical reliability of these two processed EEG methodologies. Although the increased prevalence of prolonged episodes of discordant SE was not sufficiently high to affect the overall statistical comparison between SE and BIS, the frequency of such elevations is substantive and may consequently affect clinical decisionmaking.

Importantly, the selection of our discordance threshold is not intended to imply there is no risk of awareness if the BIS or SE value remains $\leq 70$ or that this value is an appropriate target during GA. Rather, we chose this as a 
threshold above which there would be near universal concern about the possibility of awareness. Index values $>$ 70 where the end-tidal sevoflurane concentration was $>$ $1.5 \%$ thus represent a discordant elevation, as it is highly implausible that patients would either be awake or responsive to commands at this level of anesthesia.

\section{Study limitations}

The study was conducted at two different academic institutions, with only one of the two EEG monitors in use at each. Thus, a crossover analysis was not possible. Although the propensity matching by age and ASA-PS resulted in reasonably equivalent groups, there may have been unappreciated differences. Nonetheless, the large sample sizes (3,690 patients at each hospital) mitigate against this as an important confounder.

Our study only included adults $\geq 18$ yr old, so the results may not apply to pediatric patients. Because the fourth quartile of age started at $69 \mathrm{yr}$, the differences may not apply for patients of extreme age. Also, conclusions are limited to stable end-tidal sevoflurane concentrations $>$ $1.5 \%$; we do not know if results would be the same for lower concentrations. We also could not determine from our data if there were differences between SE and BIS during transitions between different sevoflurane concentrations because such determination would require simultaneous recording with both devices in the same patients. Nonetheless, given the different sampling windows and algorithmic differences between BIS and SE, it is likely that concordance would be less than at stable sevoflurane concentrations.

We were unable to study the response of the providers at $\mathrm{UMH}$ to the discordant values of SE because retrospectively discerning such behaviour from the anesthesia record would have been unreliable. We searched all free text fields in the 3,690 anesthesia records at UMH for any instances of the words "entropy" or "BIS" or "EEG" or "SE" or "RE" and found very few written comments. Also, there are often considerable delays in charting the times of drug administration to the times of administration, ${ }^{38}$ making inferences related to the timing of treatment suspect. Thus, we could not reliably determine, in retrospect, the reason for treatment or non-treatment of an elevated SE during intervals where the values appeared to be discordant.

We did not study pure intravenous anesthesia (e.g., propofol infusion with an opioid) for reasons cited in the Methods section. Anecdotally, we have noted similar frequent discordant elevations during SE but not BIS monitoring during these cases. Nonetheless, the results of our study should not be extrapolated beyond GA with a volatile agent.
Raw EEG waveforms were not captured by the AIMS, precluding systematic analysis for the presence of EMG activity. Nonetheless, when the patient appears to be adequately anesthetized, based on volatile agent end-tidal gas concentrations and lack of evidence of sympathetic responsiveness, we recommend viewing the raw EEG waveform for the presence of high-frequency EMG activity as part of the differential diagnosis. The ability to distinguish EMG activity in the displayed waveform will depend on the magnitude and frequency of the signal and the provider's experience in interpreting raw EEG. Configuring the patient monitor to display the raw EEG may be required, as this is not a default setting.

Finally, because of sample size considerations and the retrospective nature of our analysis, our study could only address the prevalence of elevated discordant values of BIS or SE, not the prevalence of depressed discordant index values in the presence of awareness. ${ }^{39}$ The recent study by Schuller et al. showing marked reduction in the BIS in 11 healthy volunteers receiving paralytic doses of succinylcholine or rocuronium, in the absence of any anesthetic or amnestic agents, suggests caution in the interpretation of BIS during deep neuromuscular blockade. ${ }^{40}$ Such considerations may be less likely with SE because the EMG signal is largely filtered by the entropy algorithm ${ }^{3}$; thus, removing the EMG entirely through neuromuscular blockade should not falsely depress the SE. The potential effects of EMG activity on SE and BIS suggest greater attention to the EMG signal is required when interpreting processed EEG indices.

\section{Conclusions}

There is a considerable prevalence of elevated discordant SE values during GA with sevoflurane that could potentially affect the behaviour of clinicians in the operating room and lead to unnecessary medication administration. Similar elevated discordant BIS values also can occur, ${ }^{41}$ but more than an order of magnitude less frequently than with SE. Providers using SE or BIS during GA to titrate the depth of anesthesia need to assess whether a high EEG index value truly reflects a hypnotic state, placing the patient at risk for intraoperative awareness, or is simply the result of the device's processing algorithm. Evaluation of the volatile agent concentrations, consideration of vital signs and patient movement, and examination of the raw EEG for the presence of EMG activity are actions to consider rather than reflexively reacting to the EEG index number displayed on the screen.

Conflicts of interest None declared. 
Editorial responsibility This submission was handled by Dr. Hilary P. Grocott, Editor-in-Chief, Canadian Journal of Anesthesia.

Author contributions Richard H. Epstein, Joni M. Maga, Michael E. Mahla, Eric S. Schwenk, and Marc J. Bloom contributed substantially to all aspects of this manuscript, including conception and design, acquisition and interpretation of data, and drafting the article.

Funding This research did not receive any specific grant from funding agencies in the public, commercial, or not-for-profit sectors.

\section{References}

1. Palanca BJ, Mashour GA, Avidan MS. Processed electroencephalogram in depth of anesthesia monitoring. Curr Opin Anaesthesiol 2009; 22: 553-9.

2. Punjasawadwong $Y$, Phongchiewboon A, Bunchungmongkol N. Bispectral index for improving anaesthetic delivery and postoperative recovery. Cochrane Database Syst Rev 2014; 6: CD003843.

3. Bruhn J, Lehmann LE, Röpcke H, Bouillon TW, Hoeft A. Shannon entropy applied to the measurement of the electroencephalographic effects of desflurane. Anesthesiology 2001; 95: 30-5.

4. Viertiö-Oja H, Maja V, Särkelä $M$, et al. Description of the entropy algorithm as applied in the Datex-Ohmeda S/5 Entropy Module. Acta Anaesthesiol Scand 2004; 48: 154-61.

5. GE Healthcare. Entropy. Available from URL: http:// clinicalview.gehealthcare.com/download.php?obj_id=318 (accessed November 2017).

6. Kelley SD. Monitoring Consciousness - Using the Bispectral Index ${ }^{\mathrm{TM}}$ (BIS $^{\mathrm{TM}}$ ) During Anesthesia. Available from URL: http:// www.covidien.com/imageServer.aspx/doc252087.pdf?contentID= 77508\& contenttype $=$ application/pdf (accessed November 2017).

7. Flaishon $R$, Windsor A, Sigl J, Sebel PS. Recovery of consciousness after thiopental or propofol. Bispectral index and the isolated forearm technique. Anesthesiology 1997; 86: 613-9.

8. Aho AJ, Yli-Hankala A, Lyytikäinen LP, Jäntti V. Facial muscle activity, response entropy, and state entropy indices during noxious stimuli in propofol-nitrous oxide or propofol-nitrous oxide-remifentanil anaesthesia without neuromuscular block. Br J Anaesth 2009; 102: 227-33.

9. Pilge S, Kreuzer M, Karatchiviev V, Kochs EF, Malcharek M, Schneider $G$. Differences between state entropy and bispectral index during analysis of identical electroencephalogram signals: a comparison with two randomised anaesthetic techniques. Eur $\mathbf{J}$ Anaesthesiol 2015; 32: 354-65.

10. CONSORT. Transparent reporting of trials. Available from URL: http://www.consort-statement.org (accessed November 2017).

11. Vuyk J, Engbers FH, Lemmens HJ, et al. Pharmacodynamics of propofol in female patients. Anesthesiology 1992; 77: 3-9.

12. Milne SE, Troy A, Irwin MG, Kenny GN. Relationship between bispectral index, auditory evoked potential index and effect-site EC50 for propofol at two clinical end-points. Br J Anaesth 2003; 90: 127-31.

13. Iwakiri H, Nishihara $N$, Nagata O, Matsukawa T, Ozaki M, Sessler DI. Individual effect-site concentrations of propofol are similar at loss of consciousness and at awakening. Anesth Analg 2005; 100: 107-10.

14. Katoh T, Ikeda $K$. The minimum alveolar concentration (MAC) of sevoflurane in humans. Anesthesiology 1987; 66: 301-3.
15. Lysakowski $C$, Elia N, Czarnetzki $C$, et al. Bispectral and spectral entropy indices at propofol-induced loss of consciousness in young and elderly patients. Br J Anaesth 2009; 103: 387-93.

16. Reich DL, Hossain S, Krol M, et al. Predictors of hypotension after induction of general anesthesia. Anesth Analg 2005; 101: 622-8.

17. Wikipedia. Greedy Algorithm. Available frm URL: https://en. wikipedia.org/wiki/Greedy_algorithm (accessed November 2017).

18. Nickalls RW, Mapleson WW. Age-related iso-MAC charts for isoflurane, sevoflurane and desflurane in man. Br J Anaesth 2003; 91: 170-4.

19. Avidan MS, Zhang L, Burnside BA, et al. Anesthesia awareness and the bispectral index. N Engl J Med 2008; 358: 1097-108.

20. Katoh T, Suguro Y, Kimura T, Ikeda K. Cerebral awakening concentration of sevoflurane and isoflurane predicted during slow and fast alveolar washout. Anesth Analg 1993; 77: 1012-7.

21. Eger EI 2nd. Age, minimum alveolar anesthetic concentration, and minimum alveolar anesthestic concentration awake. Anesth Analg 2001; 93: 947-53.

22. Manyam SC, Gupta DK, Johnson KB, et al. Opioid-volatile anesthetic synergy: a response surface model with remifentanil and sevoflurane as prototypes. Anesthesiology 2006; 105: 267-78.

23. Glass PS, Bloom M, Kearse L, Rosow C, Sebel P, Manberg P. Bispectral analysis measures sedation and memory effects of propofol, midazolam, isoflurane, and alfentanil in healthy volunteers. Anesthesiology 1997; 86: 836-47.

24. Ledolter J, Dexter F, Epstein RH. Analysis of variance of communication latencies in anesthesia: comparing means of multiple log-normal distributions. Anesth Analg 2011; 113: 88896.

25. Dexter F, Epstein RH, Marcon E, Ledolter J. Estimating the incidence of prolonged turnover times and delays by time of day. Anesthesiology 2005; 102: 1242-8.

26. Dexter $F$, Epstein $R H$. Increased mean time from end of surgery to operating room exit in a historical cohort of cases with prolonged time to extubation. Anesth Analg 2013; 117: 1453-9.

27. Dexter F, Marcon E, Epstein RH, Ledolter J. Validation of statistical methods to compare cancellation rates on the day of surgery. Anesth Analg 2005; 101: 465-73; Erratum: Anesth Analg 2012; 114: 693.

28. Dexter $F$. High-quality operating room management research. $\mathrm{J}$ Clin Anesth 2014; 26: 341-2.

29. Austin TM, Lam HV, Shin NS, Daily BJ, Dunn PF, Sandberg WS. Elective change of surgeon during the OR day has an operationally negligible impact on turnover time. J Clin Anesth 2014; 26: 343-9.

30. Wikipedia. Central limit theorem. Available from URL: https:// en.wikipedia.org/wiki/Central_limit_theorem (accessed November 2017).

31. $R$ Core Team. R: The $\mathrm{R}$ Project for Statistical Computing. $\mathrm{R}$ Foundation for Statistical Computing. Available from URL: http://www.R-project.org/ (accessed November 2017).

32. Munro MG. Fundamentals of electrosurgery part I: principles of radiofrequency energy for surgery. In: Feldman L, Fuchshuber P, Jones DB, editors. The SAGES Manual on the Fundamental Use of Surgical Energy (FUSE). NY: Springer Publishing; 2012. p. 26-7.

33. The National Institute for Occupational Safety and Health. EMF basics. In: Questions and Answers: EMF in the Workplace. PA: Diane Publishing; 1996: 8.

34. Dodd AZ. Spectrum allocation. In: The Essential Guide to Telecommunications. NJ: Prentice Hall Publishing; 2001: 377-8.

35. Rampil IJ. A primer for EEG signal processing in anesthesia. Anesthesiology 1998; 89: 980-1002.

36. Vakkuri A, Yli-Hankala A, Talja $P$, et al. Time-frequency balanced spectral entropy as a measure of anesthetic drug effect 
in central nervous system during sevoflurane, propofol, and thiopental anesthesia. Acta Anaesthesiol Scand 2004; 48: 145-53.

37. Pilge $S$, Schnieder G. BIS and state entropy of the EEG comparing apples and oranges. Br J Anaesth 2015; 115: 164-6.

38. Epstein RH, Dexter F, Ehrenfeld JM, Sandberg WS. Implications of event entry latency on anesthesia information management decision support systems. Anesth Analg 2009; 108: 941-7.

39. Myles PS, Leslie K, McNeil J, Forbes A, Chan MT. Bispectral index monitoring to prevent awareness during anaesthesia: the BAware randomized controlled trial. Lancet 2004; 363: 1757-63.
40. Schuller PJ, Newelll S, Strickland PA, Barry JJ. Response of bispectral index to neuromuscular block in awake volunteers. Br J Anaesth 2015; 115(Suppl 1): i95-103.

41. Panousis P, Heller AR, Burghardt M, Bleyl JU, Koch T. The effects of electromyographic activity on the accuracy of the Narcotrend ${ }^{\circledR}$ monitor compared with the bispectral index during combined anaesthesia. Anaesthesia 2007; 62: 868-74. 